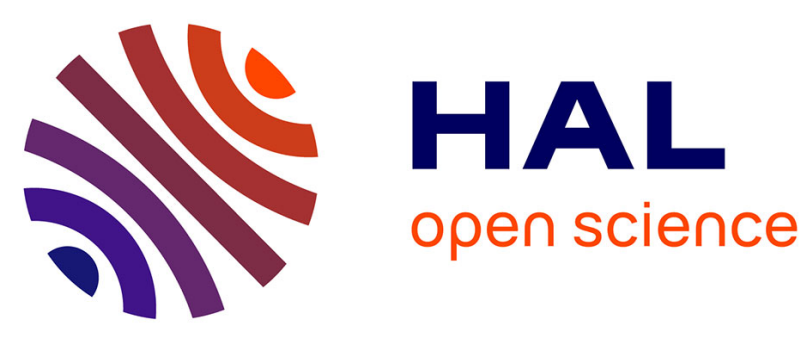

\title{
Les nouvelles technologies
}

Laurence Burgorgue-Larsen

\section{To cite this version:}

Laurence Burgorgue-Larsen. Les nouvelles technologies. Pouvoirs - Revue française d'études constitutionnelles et politiques, 2009, L'état des libertés, 130, pp.65 - 80. hal-01584313

\section{HAL Id: hal-01584313 https://hal.science/hal-01584313}

Submitted on 8 Sep 2017

HAL is a multi-disciplinary open access archive for the deposit and dissemination of scientific research documents, whether they are published or not. The documents may come from teaching and research institutions in France or abroad, or from public or private research centers.
L'archive ouverte pluridisciplinaire HAL, est destinée au dépôt et à la diffusion de documents scientifiques de niveau recherche, publiés ou non, émanant des établissements d'enseignement et de recherche français ou étrangers, des laboratoires publics ou privés. 


\section{LES NOUVELLES TECHNOLOGIES}

es nouvelles technologies de l'information et de la communication

(NTIC) sont partout. Elles ont envahi la vie personnelle des individus, transformé leurs manières de vivre, de penser, de chercher, de s'informer, de communiquer, d'échanger, de travailler et même de consommer. Dans le même temps, elles sont au cœur du développement des acteurs économiques, qu'ils soient issus de l'univers des petites et moyennes entreprises ou des multinationales les plus imposantes. En un mot, elles configurent irrémédiablement les relations de l'homme au progrès en ce début de xxI siècle. La création d'un portefeuille de Secrétaire d'État chargé de la prospective et du développement de l'économie numérique ${ }^{1}$ démontre la transformation de la société française, qui ne peut passer à côté de la révolution technologique qu'incarne l'internet ${ }^{2}$. Bien que l'image du réseau soit celle qui vienne spontanément à l'esprit pour le représenter, il s'agit en réalité d'un "protocole commun à tous les ordinateurs et susceptible d'emprunter indifféremment de nombreux réseaux: téléphone, système interne d'entreprise, télévision par câble, satellite, fibre optique ${ }^{3} \ldots$ ». Cette révolution numérique vit déjà une deuxième génération avec l'apparition de ce que les spécialistes appellent l'internet 2.0 - l'internet collaboratif ou participatif - qui « repose sur l'augmentation de la capacité de traitement des ordinateurs et du débit de connexion des foyers qui permettent de travailler en temps réel sur

1. Il a (déjà) été occupé par deux titulaires, Éric Besson tout d'abord, puis Nathalie Kosciusko-Morizet ensuite. Le premier a même écrit un essai qui prend l'allure d'un éloge des nouvelles technologies, La République numérique, Grasset, 2009.

2. L'Académie française (www.academie-francaise.fr) recommande de le nommer avec l'article et sans la majuscule à l'instar du téléphone, de l'électricité ou encore de la télévision. C'est dans la neuvième édition du Dictionnaire de l'Académie que le mot internet a été intégré.

3. Joël de Rosnay, "Ce que va changer la révolution informationnelle ", Le Monde diplomatique, $\mathrm{n}^{\circ} 521,1997$, p. 28.

POUVOIRS - 130.2009 
des bases partagées ${ }^{4}$ ». Autant dire que les progrès à venir sont encore nombreux et que nous ne sommes qu'au début d'une ère communicationnelle profondément nouvelle. On sait bien toutefois que chaque progrès technologique apporte son lot de bienfaits et de nuisances; l'internet n'échappe pas à la règle, et bien qu'il recèle, ainsi que nous le montrerons, des vertus émancipatrices, il engendre aussi des attitudes tyranniques. Car l'homme qui l'utilise reste lui-même: comme en tous domaines, il est l'artisan du meilleur comme du pire.

\section{L'ÉMANCIPATION TECHNOLOGIQUE}

Il n'est pas aberrant aujourd'hui de considérer que l'individu - très souvent en perte de repères civiques - est en mesure de se réapproprier son statut de citoyen avisé, informé, éduqué, grâce aux nouvelles technologies et plus particulièrement grâce à l'internet. Ce mode de communication lui donne en effet les moyens d'une émancipation cognitive et démocratique.

\section{L'émancipation cognitive}

Le savoir est émancipateur tandis que l'ignorance est avilissante: l'histoire de l'humanité pourrait se résumer à cette formule. Or, l'internet métamorphose radicalement le rapport au savoir de tous les individus, des plus fortunés aux plus indigents, des mieux éduqués aux plus incultes. Ce que propose cet outil numérique - sorte de passeur, un passeur de savoir -, c'est l'accès instantané à la connaissance dans tous les domaines. Une bibliothèque géante et universelle, en somme, déterritorialisée mais formidablement accessible. En plus des encyclopédies numériques qui ont fait florès dans tous les domaines - de l'encyclopédie générale et interactive Wikipédia aux plus spécifiques et militantes comme Kabylpédia (qui promeut la langue et la culture berbères) en passant par les plus pointues lancées et alimentées par des spécialistes (comme l'Encyclopédie de la vie qui recense les espèces vivantes) -, on y trouve également la Bibliothèque numérique mondiale $\left(\mathrm{BNM}^{5}\right)^{5}$. Ordonner et rendre accessible le savoir était l'ambition des encyclopédistes des Lumières, elle l'est plus que jamais pour l'UNEsCO, qui fut le maître d'œuvre de ce projet pharaonique imaginé par le directeur de la très prestigieuse Bibliothèque du Congrès américain. Quatre ans après sa conception,

4. Éric Besson, La République numérique, op.cit., p. 35.

5. Le 21 avril 2009 , jour du lancement du site de la BNM par l'UNESCO, deviendra sans doute une date historique: voir le site www.wdl.org/fr. 
le projet est devenu une réalité tangible. L'ambition est importante: pouvoir naviguer - tel un explorateur des temps modernes, c'est-à-dire à partir de chez soi, d'une bibliothèque, d'un cybercafé, bref à partir d'une connexion à l'internet - au sein des plus grandes bibliothèques du monde. Les trésors cartographiques et littéraires de la Library of Congress de Washington, du Centre d'étude et d'histoire du Mexique, en passant par la Bibliothèque universitaire de Prétoria ou encore la Bibliothèque nationale de France (entre autres) sont désormais accessibles en temps réel partout dans le monde. Cette bibliothèque numérique complète d'autres démarches lancées par Google - qui a déjà numérisé 7 millions d'ouvrages - ou par l'Union européenne qui avec Europeana ${ }^{6}$ et 4,6 millions d'ouvrages numérisés entend, elle aussi, participer à cette révolution cognitive. Il n'est plus une société savante ou un institut de recherche prestigieux qui ne dispose de sa page web sur laquelle l'accès au savoir ne soit pas organisé avec la possibilité de télécharger des textes d'articles et d'interviews, mais aussi d'écouter des émissions culturelles et scientifiques grâce à la technologie du Podcast ${ }^{7}$. Comment ne pas mentionner également que les universitaires ont su, un peu partout de par le monde mais avec des degrés variables, s'adapter en créant des cyber-revues et/ou en mettant en ligne des articles consultables partout et par tous. Autant d'éléments désormais incontournables de la diffusion des travaux de recherche et, par voie de conséquence, de la renommée des chercheurs et de leurs universités de rattachement. La révolution du savoir a, par la force des choses, entraîné des bouleversements majeurs dans le rapport au travail des individus (ainsi du télétravail), mais également les modes de fonctionnement des acteurs privés ${ }^{8}$ comme des institutions au service de la chose publique 9 . Que dire du «quatrième pouvoir", qui voit ses fondamentaux métamorphosés par l'irruption de la technique numérique: c'est tout à la fois la déontologie et les méthodes de travail des journalistes qui sont revisitées ${ }^{10}$.

6. www.europeana.eu/portal/

7. Parmi beaucoup d'autres, on renvoie au site du Collège de France (www.college-defrance.org) et à celui de l'Académie des sciences morales et politiques (www.asmp.fr).

8. Les entreprises ont ainsi lancé les achats et les paiements en ligne, autant de transactions censées être sécurisées.

9. L'exemple du monde de la justice, confronté désormais à la dématérialisation des procédures, est édifiant; voir Gérard Savater, « Nouvelles technologies et système judiciaire. Le déploiement de la communication dans les juridictions judiciaires", JCP, édit. gén., 17 décembre 2008, p. 17-21.

10. Christine Leteinturier, «De la Remington à internet, les mutations du cadre technique des journalistes », Clés pour le siècle, Dalloz, 2000, p. 993-1009. 
Ce bouleversement de l'accès au savoir ainsi que des modes de travail n'étreint certes pas tous les pays et les individus de la planète de manière identique. $\mathrm{La}$ «fracture numérique » est une donne impossible à éluder. Le niveau de vie des pays agit logiquement sur les possibilités d'accès et d'utilisation de l'internet et, ce faisant, sur l'accès au savoir universel. L'Union internationale des télécommunications a créé un nouvel indice de développement des nouvelles technologies, qui a permis de comparer l'évolution des NTIC dans 154 pays sur une période de cinq ans (20022007) ${ }^{11}$. Les résultats - édifiants - étaient prévisibles: les pays d'Europe du Nord restent en tête, tandis que les pays les moins avancés sont encore loin d'un accès et d'une utilisation généralisés. Il n'en reste pas moins que tout est fait - il s'agit d'ailleurs d'un des objectifs de la Bibliothèque numérique mondiale - pour réduire cette fracture et rendre véritablement universelle cette révolution cognitive.

\section{L'émancipation démocratique}

La représentation démocratique est malade depuis longtemps; la France ne fait pas figure d'exception sur ce point ${ }^{12}$. Même si la démocratie représentative est irremplaçable ${ }^{13}$, elle peut - beaucoup pensent qu'elle doit - être complétée par des formes de démocratie participative. C'est le credo politique du moment. Mode passagère ou bouleversement radical des mœurs politiques et du rapport des citoyens à la res publica? De quelle manière ce slogan de la postmodernité libérale peut-il concrètement se transformer en réalité positive et effective? Comment faire en sorte que la démocratie participative - qui de simple principe est devenue une valeur référentielle - soit efficace, en mesure de dégager des mécanismes procéduraux précis; bref, comment éviter qu'elle ne serve d'alibi à une simple "démocratie d'opinion»? À cela, pas de réponse évidente. Ce qui est sûr, c'est que beaucoup de branches du droit sont

11. Cet indice est établi sur la base de onze indicateurs qui, ensemble, constituent une référence aux niveaux mondial, régional et national. Ces indicateurs concernent l'accès aux nouvelles technologies, leur utilisation, les compétences dans ce domaine, ainsi que le nombre de ménages ayant un ordinateur, le nombre d'internautes et les taux d'alphabétisation.

12. Slobodan Milacic, «La démocratie représentative devant un défi historique ", in Rafaâ Ben Achour, Jean Gicquel, Slobodan Milacic (dir.), La Démocratie représentative devant un défi bistorique, Bruxelles, Bruylant, 2006, p. 1-37.

13. La démocratie représentative - irremplaçable, on insiste - est également saisie par l'internet. On renvoie ici aux délicates questions posées par le vote électronique; voir Jean Gicquel, «Le vote électronique en France», $L P A, 6$ avril 2006, $n^{\circ} 68$, p. 7; Marie de Cazals, "La dématérialisation du vote: un nouvel horizon pour la démocratie représentative? ", $R D P$, $\mathrm{n}^{\circ} 1,2008$, p. 185-210. 
imprégnées de ce questionnement et $\mathrm{y}$ apportent des réponses sectorielles. Du droit administratif de l'environnement ${ }^{14}$, en passant par le droit de la régulation économique ${ }^{15}$ jusqu'au droit constitutionnel de l'Union européenne ${ }^{16}$, la participation des citoyens à l'élaboration et à la prise de décision s'impose toujours un peu plus. Dans ce contexte, les nouvelles technologies de l'information constituent les vecteurs idéaux de la manifestation de cette participation citoyenne. En France, la campagne référendaire sur le projet de Constitution européenne a montré l'importance du Net dans la diffusion des arguments des partisans du non qui ont investi la Toile et ont fini par faire valoir et prédominer leur point de vue. $\mathrm{La}$ "netcampagne» naissait avec la manifestation d'un militantisme numérique subversif. Face à des médias classiques, jugés trop partisans - étant quasiment tous favorables à l'adoption du traité établissant une Constitution pour l'Europe -, la vague contestatrice a déferlé avec une force inouie emportant sur son passage les avancées incontestables d'un traité mal nommé, source de confusions et prétexte aux instrumentalisations politiques les plus variées ${ }^{17}$.

Cet épisode a fait comprendre aux acteurs politiques français qu'ils ne pouvaient pas rester en dehors de la mouvance numérique. La communication politique est aujourd'hui saisie à bras-le-corps par l'internet. Les récentes campagnes électorales présidentielles en France, mais surtout aux États-Unis, ont montré que les politiques, afin de n'être pas dépassés par ce nouvel outil, étaient dans l'obligation vitale de le maîtriser pour mieux l'utiliser; certains diront l'instrumentaliser. La victoire de Barak Obama, déjà surnommé le premier "web-président» de l'histoire américaine, est le résultat d'une stratégie exceptionnelle où la maîtrise technique

14. Marie-France Delhoste, $\propto$ Démocratie participative : de l'échec de l'organisation étatique à l'avenir du projet européen ", RFDA, 2007, p. 1061.

15. Martine Lombard, "Institutions de régulation économique et démocratie participative ", AJDA, 2005, p. 530.

16. On sait que le traité établissant une Constitution pour l'Europe avait jeté les bases pour octroyer une substance juridique à ce concept; voir Constance Grewe, «Article I-47 ", in Laurence Burgorgue-Larsen, Anne Levade, Fabrice Picod (dir.), Traité établissant une Constitution pour l'Europe. Commentaire article par article, Partie I et IV, Architecture constitutionnelle, Bruxelles, Bruylant, 2007, p. 624-633. Le traité sur l'Union européenne, tel qu'adopté à Lisbonne le 13 décembre 2007, reprend cette disposition à l'article 11 TUE.

17. Cet épisode impose de repenser l'appréhension de la démocratie dans le contexte européen. Pour un point de vue engagé et critique, voir Laurence Burgorgue-Larsen, «La démocratie au sein de l'Union européenne. De la "constitution composée" à la "démocratie composée" ", in Hartmut Bauer, Christian Callies (éd.), Les Principes constitutionnels en Europe. Constitutional principles in Europe, Societas Iuris Publici Europaei (SIPE), Bruylant, Sakkoulas, Berliner, 2008, p. 83-99. 
du web - via son site officiel ${ }^{18}$-, comme l'utilisation à outrance de la téléphonie mobile (envoi de millions de SMS aux internautes citoyens) ont été les atouts d'une campagne politique hors du commun. Qu'on l'accepte ou non, le fait est là: l'internet est entré dans les mœurs politiques et revisite drastiquement l'expression démocratique. Mieux, il est incroyablement subversif dans les sociétés verrouillées, tyrannisées, exploitées, encadrées par des régimes autoritaires qui entendent faire taire les voix dissidentes. $\mathrm{La}$ Chine, la Birmanie ou encore l'Iran sont des États qui entendent surveiller la Toile, les échanges et les prises de position qui s'y expriment. A contrario, il y a bien là la démonstration de sa force d'expression démocratique...

Tout serait-il parfait dans l'univers des nouvelles technologies en expansion? Poser la question, c'est évidemment apporter un élément de réponse. Toute avancée - surtout en matière de progrès scientifique - a toujours, inéluctablement, sa face sombre. Si l'internet est émancipateur, il est aussi à sa manière, de façon concomitante, tyrannique.

\section{LA TYRANNIE TECHNOLOGIQUE}

Les nouvelles technologies induisent une implosion des anciens us et coutumes fondés sur le respect de l'intimité, tandis qu'elles engendrent une aspiratic: dévorante à une liberté d'accès absolue aux données de toutes sortes. Les principes de transparence et de gratuité sont, à certains égards, les tyrannies des temps modernes contre lesquelles - équilibre précaire, sans cesse à retrouver - il faut être en mesure de se protéger.

\section{La tyrannie de la transparence}

Si l'internet révolutionne en permanence les mœurs de bon nombre de sociétés en permettant un accès généralisé au savoir ainsi qu'une nouvelle approche démocratique, il met également à mal, de façon paroxystique, certains droits et libertés. Les dérives langagières y sont coutumières, tandis que la mise à nu de l'intimité y est débridée. La liberté qu'engendre

18. www.barakobama.com. Barak Obama a ouvert son site de campagne vingt et un mois avant l'élection. À peine candidat à l'investiture du parti démocrate, il contactait Chris Hughes (25 ans), l'un des fondateurs de Facebook pour l'aider à mettre en place son site officiel d'information (programme du candidat, télévision en ligne, argumentaires, photos, vidéos, boutique, etc.). Cette stratégie a permis de recruter de nouveaux sympathisants (module viral), de collecter des fonds (le bandeau rouge Donate s'affiche sur toutes les pages) et de remonter de l'information de la «base». Pour toucher les jeunes, le candidat a également acheté de la publicité sur XBox Live dans des jeux vidéo en ligne. Autant d'éléments tout à fait inédits dans le cadre d'une campagne politique. 
ce moyen de communication est telle que la transparence des opinions (y compris les plus détestables) et de l'intimité (y compris la plus crue) est devenue l'emblème d'un monde où la technique aurait réussi à prendre l'homme au piège de sa créativité technologique. L'atteinte aux droits qui découle d'une utilisation exagérée de la Toile impose des réponses adaptées. D'une part, il faut pouvoir agir sur les dérives en matière de diffusion de propos illicites: la liberté d'expression doit être encadrée, pour ne pas dire limitée. D'autre part, la vie privée, tant dans ses aspects personnels que professionnels ${ }^{19}$, doit être préservée des multiples types d'ingérences qui ne proviennent plus des seules autorités étatiques mais en réalité, le plus souvent, des particuliers eux-mêmes (personnes physiques ou morales).

\section{La transparence des opinions}

L'irruption de l'internet - qui permet à quiconque de s'exprimer sans entraves aucune, à tout le moins avec une facilité édifiante - a logiquement engendré des dérives. Pour ceux qui entendent abuser du droit d'expression, il est très vite apparu comme le réseau communicationnel rêvé ${ }^{20}$. Les négationnistes en tout genre ou les coutumiers de l'injure ou de la diffamation n'ont pas manqué d'instrumentaliser ce moyen moderne de communiquer pour participer à la démultiplication de leurs extrémismes en temps réel sur le globe. Comment, dans ce cadre, contrer les abus sans tomber dans le même temps dans un ordre moralement correct où aucune idée qui « heurte, choque ou inquiète ${ }^{21}$ » ne serait tolérée ? Faut-il édifier un droit ad hoc, spécifique au "réseau des réseaux», et y réprimer plus durement ou au moins de façon spécifique les abus ou s'en tenir aux canons du droit de la liberté d'expression patiemment édifiés indépendamment du média en cause? Le juge suprême américain répondait à

19. La construction jurisprudentielle de la Cour européenne est imposante en la matière et peut se décliner en trois arrêts: CEDH, 30 mars 1989, Chappel c. Royaume-Uni, Série A, n ${ }^{\circ}$ 152-A; CEDH, 16 décembre 1992, Niemetz c. Allemagne, Série A, n² 251-B; CEDH, II $^{\circ}$ section, 16 avril 2002, Société Colas Est et autres c. France.

20. L'apparition de l'imprimerie a suscité les mêmes dérives, à tout le moins les mêmes craintes au Xvir siècle. Il suffit de se référer à la formule de Pierre Bayle qui, dans l'article "Dissertation sur les libellés diffamatoires» de son Dictionnaire historique et critique, 1702, vol. 4, p. 591, écrivait: "Comme il n'y a rien de si utile qui à certains égards ne cause du mal, il est arrivé que l'imprimerie, parmi cent commodités qu'elle a apportées, a donné lieu à un notable inconvénient, c'est qu'elle a fourni aux Satiriques et aux Séditieux mille moyens de répandre promptement leur venin par toute la terre."

21. Selon la célèbre formule de la Cour européenne des droits de l'homme utilisée dans l'arrêt Handyside c. Royaume-Uni du 7 décembre 1976. 
cette interrogation dès 1997 dans son important arrêt Reno ${ }^{22}$. Il affublait l'internet du plus haut degré de protection issu du Premier Amendement, déployait logiquement un contrôle strict à l'égard des ingérences dont il pouvait être l'objet, tout en considérant que la protection de la liberté d'expression variait en fonction du média considéré. Il se conformait en cela à sa jurisprudence traditionnelle ${ }^{23}$. En France, c'est le droit de la presse tel qu'issu de la loi du 29 juillet 1881 qui s'est imposé. La voie de la banalisation s'appliqua quasi naturellement. Cette solution, si elle a recélé des avantages évidents notamment quant à la définition des infractions (diffamation, atteinte à l'ordre public), n'en a pas moins dévoilé certaines difficultés qui ont tenu à la particularité de ce mode de communication. Ainsi en est-il de l'identification du point de départ du délai de prescription en matière de diffamation comme de la détermination des personnes ou institutions responsables pour les propos illicites mis en ligne. Sur ces deux derniers points, les réponses furent données par le législateur communautaire ${ }^{24}$, relayé par le législateur français ${ }^{25}$, étroitement contrôlé par le Conseil constitutionnel ${ }^{26}$. En dehors des difficultés inhérentes à l'articulation entre les ordres juridiques que cette sorte de ménage juridique à trois recèle, il est de plus en plus difficile aujourd'hui de continuer à affirmer de façon péremptoire qu'un droit spécifique n'a pas vu le jour aux fins de répression des abus effectués sur la Toile. En effet, si le droit de la presse trouve à s'appliquer à l'internet à partir du moment où l'auteur d'une publication litigieuse ou l'éditeur d'un site sont dûment identifiés, un droit spécial des intermédiaires techniques (fournisseurs d'accès et hébergeurs) existe bel et bien pour avoir favorisé la diffusion de contenus illicites ${ }^{27}$.

22. Reno, Attorney General of the United States et al. c. American Civil Liberties Union et al., 117 S.Ct 2329, 1997.

23. Anne Klebes-Pelissier, «Internet et la liberté d'expression. À propos de l'arrêt de la Cour suprême des États-Unis Reno v. American Civil Liberties Union du 26 juin 1997 », RUDH, 1998, p. 393-401.

24. Directive «commerce électronique», 8 juin 2000 (JOCE, nº 178, 17 juillet 2000, p. 1).

25. Loi sur la confiance dans l'économie numérique du 21 juin 2004 UO, 22 juin 2004, p. 11168).

26. CC, 10 juin 2004 , Économie numérique, $\mathrm{n}^{\circ} 2004-496$ DC.

27. Pour un aperçu général de la problématique, voir Luc Grynbaum, "Internet », Dic-

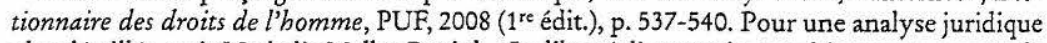
plus détaillée, voir Nathalie Mallet-Poujol, «La liberté d'expression sur l'internet: aspects de droit interne", $D, 2007$, p. 591. 


\section{La transparence de l'intimité}

Pour vivre heureux, vivons cachés. Ce proverbe attribué à Jean-Pierre Florian semble à des années-lumière des mœurs contemporaines. Être in aujourd'hui, c'est être sur la Toile, avoir son cortège d' «amis» sur Facebook - le plus fameux des sites dits «communautaires»-, y exposer ses photos et y raconter sa vie. La société est devenue impudique et les moyens modernes de communication ont été des accélérateurs de cette impudeur privée qui s'est transformée en impudeur sociale. Ces mises à nu volontaires, délibérées, revendiquées ont leurs effets pervers car l'internet a de la mémoire; le droit à l'oubli n'est certainement pas son credo ${ }^{28}$. La naïveté, l'innocence ou tout simplement l'inconscience d'un moment ou d'une période peuvent, quelques années plus tard, se transformer en fardeau. Chercher un emploi ou le garder peut être conditionné par les informations intimes que des employeurs pourront trouver sur des salariés en surfant rapidement et aisément sur le web pour in fine refuser de les embaucher ou à l'inverse décider de les licencier. À cette exposition volontaire sur la Toile avec son cortège de dérives s'ajoute l'instrumentalisation de données personnelles par des opérateurs privés à des fins multiples, qui sont en général illicites et lucratives. De la contrefaçon ${ }^{29}$ à la pédopornographie ${ }^{30}$, en passant par le piratage ou l'escroquerie ${ }^{31}$, la gamme du détournement des données personnelles est particulièrement large, au point d'avoir généré une nouvelle catégorie d'infraction - la cybercriminalité ${ }^{32}$ - et un texte de droit international qui

28. Le 28c Rapport de la CNIL expose parfaitement les difficultés qu'il y a pour faire supprimer une page web contenant des données personnelles, voir Rapport d'activité 2007, Paris, 2008 , p. 58

29. Cass., crim., 5 février 2008, req. 07-81.387. Des photographes qui, munis d'une accréditation de presse de la Fédération française de couture, prennent des clichés lors de défilés de mode qu'ils diffusent ensuite sur un site Internet sans avoir obtenu l'accord des maisons de couture, titulaires de droits d'auteurs sur leurs créations et défilés, sont coupables de contrefaçon, voir AJ Pénal, 2008, p. 236, commentaire de Guillaume Royer.

30. La pédopornographie engendre la nécessité évidente de préserver les mineurs sur la Toile, voir l'étude de Sylvia Bréger, «La protection des mineurs sur Internet ", AJ Pénal, 2009, p. 112.

31. Le phising - dont l'intitulé provient de la contraction des mots anglais fishing (pêche) et phreaking (piratage de lignes informatiques), traduit parfois par « hameçonnage » - est une technique frauduleuse utilisée par des pirates informatiques pour récupérer des informations (en général bancaires) auprès d'internautes.

32. L'Organisation des Nations unies la définit comme « tout comportement illégal faisant intervenir les opérateurs électroniques qui visent la sécurité des systèmes informatiques et des données qu'ils traitent ", tandis que le Ministère français de l'Intérieur la définit plus simplement comme «l'ensemble des infractions pénales qui se commettent sur le réseau internet " (www. interieur.gouv.fr). 
entend y faire face: la Convention sur la cybercriminalité adoptée sous l'égide du Conseil de l'Europe ${ }^{33}$. Le législateur français n'a pas manqué d'adapter l'état du droit à l'évolution de la criminalité informatique en adoptant pas moins de cinq lois après que la loi pionnière "informatique et libertés» abordait la question il y a plus de trente ans ${ }^{34}$.

Si l'intimité personnelle des individus est aujourd'hui aux prises avec les criminels du cyberespace ${ }^{35}$, l'intimité professionnelle est quant à elle mise à mal par une certaine conception des impératifs de rentabilité économique. Le salarié peut voir ses courriels épiés et in fine décryptés ou encore ses coups de fil enregistrés. Alors que Jean-Emmanuel Ray écrivait, il y a plus de dix ans, que le monde du travail était face à un vide juridique complet dans la prise en compte des nouvelles technologies de l'information ${ }^{36}$, la réalité est aujourd'hui à l'opposé de ce néant originel. L'inflation législative et jurisprudentielle est à son comble; le législateur puis le juge sont entrés dans une quête incessante du juste équilibre dans un monde où l'innovation technologique défie le temps normatif et contentieux. Dans ce contexte, les juges suprêmes - juges conventionnels de droit commun - ont à charge d'utiliser les atouts de la Convention de sauvegarde des droits et libertés fondamentales, telle qu'interprétée par le juge de Strasbourg, afin d'ajuster au mieux l'état des libertés à l'accélération technologique. Ils ne manquent pas de le faire, en étant même précurseurs dans ce domaine. La Chambre sociale de la Cour de cassation n'attendait pas l'arrêt Copland ${ }^{37}$ de la Cour européenne de

33. Conseil de l'Europe, Convention sur la cybercriminalité, STE, $\mathrm{n}^{\circ} 185$, adoptée à Budapest le 23 novembre 2001. Un protocole y était adjoint le 30 janvier 2003 relatif à l'incrimination d'actes de nature raciste et xénophobe commis par le biais de systèmes informatiques.

34. Frédérique Chopin, "Les politiques publiques de lutte contre la cybercriminalité», AJ Pénal, 2009, p. 101. L'auteur recense, depuis la loi de 1978 créant la CNIL, la loi Godfrain, la loi relative à la sécurité quotidienne, la loi pour la sécurité intérieure, la loi portant adaptation de la justice aux évolutions de la criminalité, la loi pour la confiance dans l'économie numérique, la loi relative aux communications électroniques et aux services de communication, la loi de prévention de la délinquance et la loi visant à prolonger certaines dispositions de la loi relative à la lutte contre le terrorisme.

35. La criminalité numérique prend également l'allure d'une " guerre " de déstabilisation des entreprises, des gouvernements, des associations en tous genres par l'utilisation de l'internet; voir Nicolas Arpagian, La Cyberguerre. La guerre numérique a commencé, Vuibert, 2009.

36. Jean-Emmanuel Ray, «De Germinal à Internet: une nécessaire évolution du critère du contrat de travail ", Droit social, 1995, n 7-8, p. 634-637.

37. CEDH, 3 avril 2007, Copland c. Royaume-Uni. Cet arrêt s'inscrit dans la ligne jurisprudentielle dessinée par les arrêts Klass c. Allemagne du 9 septembre 1978, Malone c. Royaume-Uni du 2 août 1984 et Halford c. Royaume-Uni du 25 juin 1997. La Cour considère dans l'affaire Copland que l'envoi de courriels depuis le lieu de travail et l'utilisation à des fins personnelles de l'internet relèvent de la protection de l'article 8, voir Jean-Pierre Marguénaud, Jean Mouly, 
2007 , pour affirmer dès 2001 dans le célèbre arrêt Nikon ${ }^{38}$ - au visa de l'article 8 de la Convention - qu'un employeur n'avait pas le droit d'ouvrir le courriel d'un salarié lorsque celui-ci titrait son courrier électronique "personnel ». Une fois les principes classiques et fondamentaux relevant de la protection de la vie privée appliqués à l'internet et aux nouveaux modes de correspondance qu'il induit, le juge n'en doit pas moins, à chaque instant, adapter ses solutions à la complexité du réel numérique et de ce que l'on appelle la cybersurveillance ${ }^{39}$.

La surveillance - qui est évidemment techniquement protéiforme n'est certainement pas le fait exclusif des entités privées. La protection de la vie privée s'est historiquement forgée en réaction aux intrusions de l'État et de ses organes dans la vie des personnes vivant sur son territoire. Le contexte politique consécutif aux attentats du 11 septembre 2001 - portant un coup majeur à l' « hyperpuissance américaine » (Hubert Védrine) - a sensiblement réactivé les réflexes sécuritaires des États. Ils ont tous adopté des lois qui portent des coups de canif importants à certains droits et plus particulièrement au droit à la vie privée. Dans cette dialectique infernale de lutte contre le terrorisme et de protection des droits fondamentaux, les juges constitutionnels et/ou suprêmes de chaque pays tentent (exercice éminemment complexe) de trouver, une fois encore, le juste équilibre ${ }^{40}$ : La mise en fiche des individus est également

* Les incursions de la Cour européenne des droits de l'homme en droit du travail: une œuvre encore en demi-teinte ", Revue du droit du travail, 2008, p. 16 sq.

38. Cass., soc., 2 octobre 2001, Société Nikon France c. Frédéric Onof, req. 99-42.942, Bull. civ., $\mathrm{n}^{\circ} 291$.

39. Dans un arrêt de la Chambre commerciale de la Cour de cassation du 15 mai 2007, Société $G M B A$, il est apporté un tempérament à la jurisprudence Nikon conformément aux exigences tirées de l'article 6 de la Convention. Ainsi, « toute atteinte à la vie privée n'est pas interdite et une telle atteinte peut être justifiée par l'exigence de la protection d'autres intérêts, dont celle des droits de la défense, si elle reste proportionnée au regard des intérêts antinomiques en présence». De même, dans un arrêt de la Chambre sociale de la Cour de Cassation du 9 juillet 2008, Entreprise Martin, le juge judiciaire généralise en quelque sorte le droit d'accès de l'employeur sur l'historique de la navigation de chaque salarié: «Les connexions établies par un salarié sur des sites internet pendant son temps de travail grâce à l'outil informatique mis à sa disposition par son employeur pour l'exécution de son travail sont présumées avoir un caractère professionnel, de sorte que l'employeur peut les rechercher aux fins de les identifier, hors de sa présence."

40. On se reportera au très étonnant et intéressant arrêt de la Cour constitutionnelle allemande du 27 février 2008 (BVerfG 1 BvR 370/07 et 1 BvR 595/07). Le juge constitutionnel allemand a considéré que deux mesures introduites par le législateur constitutionnel du Land de Nordrhein-Westfalen, qui avaient pour objet d'autoriser la fouille secrète des ordinateurs par le décryptage des communications et leur surveillance à distance dans le contexte de lutte contre le terrorisme, étaient inconstitutionnelles. Le fondement d'une telle solution ne passa 
une tendance lourde, contre laquelle les autorités indépendantes doivent constamment lutter ${ }^{41}$. La surveillance (indépendante) de la surveillance (étatique) est vitale. En France, la Commission nationale informatique et libertés (CNIL) a une mission de contrôle ${ }^{42}$ et de sanction ${ }^{43}$ existentielles qu'elle exerce avec une indépendance remarquable ${ }^{44}$. À l'échelle européenne, le " G29 », mis en place par la directive 95/46/CE du 24 octobre 1995 sur la protection des données à caractère personnel, détient une mission stratégique: contribuer à élaborer des règles européennes en matière de protection des données personnelles ${ }^{45}$. Autant de questions qui, mondialisation oblige, nécessitent des réponses à l'échelle européenne et internationale ${ }^{46}$.

\section{La tyrannie de la gratuité}

Avoir accès à tout. Avoir droit d'accéder gratuitement à toutes les œuvres artistiques (musicales et audiovisuelles notamment) est la revendication mondiale du moment. La circulation automatisée et facilitée à l'information

pas inaperçu: «Le Tribunal constitutionnel choisit l'article 2, alinéa 1, protégeant le libre épanouissement de la personnalité, combiné avec l'article 1 alinéa 1 consacrant l'intangibilité de la dignité humaine. Il développe à partir de ces dispositions une nouvelle expression du droit général de la personnalité (allgemeines Persönlichkeitsrecht) qu'il désigne sous le terme de «droit fondamental à la garantie de la confidentialité et de l'intégrité des systèmes techniques d'information" (Grundrecht auf Gewäbrleistung der Vertraulichkeit und Integrität informationstechnischer Systeme) ", voir Juliette Lelieur, Claire Saas, "Chronique de droit pénal constitutionnel allemand 2007-2008 ", Revue de science criminelle et de droit pénal comparé, 2008, p. 676; d'une manière générale, sur le traitement du concept de dignité en Allemagne, voir Luc Heuschling, "La dignité de l'être humain dans la jurisprudence constitutionnelle allemande", in Laurence Burgorgue-Larsen (dir.), La Dignité saisie par les juges, Bruxelles, Bruylant (à paraître).

41. Sur cette tendance qui se manifeste également à l'échelle de l'Union européenne, on renvoie à l'article très fouillé de Jean-Marie Delarue, «L'Europe des fichiers. Dialogue des juges, des policiers, des autorités administratives indépendantes ", Le Dialogue des juges. Mélanges en l'bonneur du président Genevois, Dalloz, 2009, p. 233-304.

42. La rapidité de l'évolution technologique pose toutefois la question de la pertinence du mode de fonctionnement de la CNIL dans le cadre des techniques de contrôle préalable. On se reportera à l'analyse comparée entre la France et la Suède sur la question; voir Patricia BlancGonnet Jonason, «Vers une meilleure adaptation du droit de la protection des données personnelles à la réalité informationnelle ", Actualité juridique de droit administratif, 2008, p. 2105.

43. CNIL, délibération n $n^{\circ} 2008-163$ du 12 juin 2008 prononçant une sanction pécuniaire à l'encontre de la société Neuf Cegetel.

44. Ad ex., délibération 2008-174 du 16 juin 2008 portant avis sur un projet de décret en Conseil d'État portant création au profit de la Direction centrale de la sécurité publique d'un traitement automatisé de données à caractère personnel dénommé «Edvige».

45. Le nom du groupe découle de la base juridique qui en est à l'origine, à savoir l'article 29 de la directive de 1995. C'est le président de la CNIL, Alex Türck, qui depuis le 19 février 2008 assure la direction des travaux du « G29 ».

46. Voir les travaux de la Conférence mondiale des autorités de protection des données. 
a induit, partout sur la planète, une volonté considérée comme légitime et naturelle de pouvoir, à tout instant, grâce à un simple clic informatique, télécharger vidéos, musiques et films au gré des envies, des passions, des goûts d'individus qui estiment avoir le droit de s'approprier et de s'échanger des œuvres créées récemment. Et les droits d'auteurs des artistes interprètes dans cette affaire? Les temps modernes sont ainsi faits que la plupart des internautes n'en ont cure, eux qui estiment que la culture doit rester en dehors de la logique marchande. Les débats les plus passionnés se manifestent dès qu'il est question de mettre de l'ordre dans cette douce anarchie des mœurs numériques ${ }^{47}$. Une interrogation suffira à synthétiser les enjeux. Comment arriver à préserver les légitimes droits d'auteurs ${ }^{48}$ sans mettre en place une société de surveillance et de répression judiciaire massive qui, en plus d'être susceptible de porter atteinte à un autre droit fondamental (la vie privée), deviendrait au regard de la fulgurance sans cesse renouvelée des progrès technologiques une option très vite dépassée ? Équilibre complexe où le juge communautaire a été amené à donner le la. La Cour de justice est effectivement entrée dans la partie grâce aux ressorts toujours bienfaisants du mécanisme préjudiciel ${ }^{49}$. Dans une importante affaire qui opposait à l'instance une association espagnole de producteurs de musique (Promusicae) au géant européen de la téléphonie et de l'internet (Telefónica $S A$ ), la Cour, après une analyse des différentes directives qui protégeaient des droits en conflit in casu, a considéré que le droit communautaire positif n'imposait pas aux États membres de "prévoir [...] l'obligation de communiquer des données à caractère personnel en vue d'assurer la protection effective du droit d'auteur dans le cadre d'une procédure civile». Les internautes accoutumés au téléchargement et à l'échange des œuvres peuvent se sentir à leur aise car la demande de Promusicae au juge national consistait

47. Pour un point de vue engagé de l'ancien PDG de la FNAC, Denis Olivennes, La gratuité, c'est le vol. Quand le piratage tue la culture, Grasset, 2007. C'est sur la base du rapport qu'il a remis au ministre de la Culture, Christine Albanel, que le projet de loi dit «Hadopi » ou «Création et internet» a été préparé par le gouvernement de François Fillon.

48. Les droits de propriété intellectuelle, tel le droit d'auteur, font partie du droit de propriété et constituent, pour la Cour de justice des Communautés européennes, des principes généraux du droit communautaire, CJCE, 12 septembre 2006, Laserdisken, C 479/04, Rec. p. I-8089, point 65. La jurisprudence constitutionnelle est en osmose parfaite avec celle de la Cour de Luxembourg: CC, 27 juillet 2006, Loi relative aux droit d'autenr et aux droits voisins, $n^{\circ}$ 2006-540 DC. Autant d'avancées qui ravissent les privatistes qui estiment que l'unification du régime général des biens était essentielle; voir Thierry Revet, «Les droits de propriété intellectuelle sont des droits d'auteur ", RTD Civ., 2006, p. 791.

49. CJCE, grande ch., 29 janvier 2008, Promusicae SA, C-275/06; conclusions de l'avocat général Juliane Kokott prononcées le 18 juillet 2007. 
à ordonner à Telefónica, dans le cadre d'une procédure judiciaire, que soient relevées l'identité et l'adresse physique des personnes qui utilisent des programmes d'échange d'archives, communément appelé peer to peer ${ }^{50}$. Cette non-obligation de divulgation laisserait croire que la protection des données personnelles l'emporterait sur la préservation des droits d'auteur. Pas tout à fait. Car le juge communautaire, conscient des enjeux en présence et des équilibres à préserver, exige des États deux choses. Au moment de la transposition des directives tout d'abord, les États «doivent veiller à se fonder sur une interprétation de celles-ci qui permette d'assurer un juste équilibre entre les différents droits fondamentaux protégés par l'ordre juridique communautaire». Au moment de leur mise en œuvre ensuite, «il incombe aux autorités et aux juridictions des États membres non seulement d'interpréter leur droit national d'une manière conforme à ces mêmes directives, mais également de ne pas se fonder sur une interprétation de celles-ci qui entrerait en conflit avec lesdits droits fondamentaux ou avec les autres principes généraux du droit communautaire, tels que le principe de proportionnalité». Obscure clarté communautaire ${ }^{51}$ ? Loin s'en faut. Le juge de Luxembourg renvoie la balle dans le camp interne, où législateurs et juges devront chacun à leur niveau assurer le nécessaire équilibre entre la préservation des droits d'auteur et la protection de la vie privée à travers la confidentialité des données personnelles. Cette marge d'appréciation est bienvenue: elle laisse aux instances nationales, conformément à leur sensibilité, le soin de déterminer les équilibres. Dans le même temps, la diversité qu'elle induit va certainement générer des perturbations sur le marché de l'internet. Si la France entend opter pour un «système gradué» de sanction avec la loi Création et internet ${ }^{52}$, d'autres pays sont plus radicaux, à l'instar du Danemark, de la Hollande, de la Finlande, de la Slovénie, du Royaume-Uni ${ }^{53}$ et, plus récemment, de la Suède. Ce pays adoptait en

50. En l'occurrence le logiciel d'échange s'appelait $\mathrm{KaZaA}$.

51. Certains l'ont pensé et dénoncé, voir Kate Brimsted, Gavin Chesney, «The ECJ's judgement in Promusicae: The Unintended Consequences - Music to the Ears of Copyrights Owners or a Privacy Haedache for the Future? A comment ", Computer Law \& Security Report, 2008, p. 275-279.

52. Les débats passionnés ainsi que les stratégies politiques rocambolesques qui ont émaillé les discussions de la loi Création et internet en France - qui finit par être adoptée le 13 mai 2009 (296 voix pour et 233 voix contre à l'Assemblée nationale; 189 voix pour et 14 voix contre au Sénat pour être aussitôt censurée par le Conseil constitutionnel le 10 juin 2009, décision $\mathrm{n}^{\circ}$ 2008-580 DC) - sont symptomatiques des enjeux sociétaux que l'on retrouve partout en Europe et dans le reste du monde.

53. On se reportera aux informations fournies par Xavier Groussot, « Rock the KaZaA: Another Clash of Fundamental Rights ", CMLR, 45, 2008, p. 1745-1766. 
effet le 25 février 2009 une loi obligeant les opérateurs à livrer à la justice l'adresse d'un ordinateur sur lequel aurait été téléchargé illégalement un fichier ${ }^{54}$. Au bout du compte, la diversité respectueuse des États ne va-t-elle pas générer ce que d'aucuns ont déjà appelé un ISP Shopping (Internet Service Provider Shopping)? Les internautes définitivement accros au téléchargement illégal pourraient trouver un fournisseur ISP basé dans un pays où l'obligation de divulgation des données personnelles dans le cours d'une procédure judiciaire civile n'existerait pas... De la difficulté de trouver les équilibres tant au niveau de l'élaboration de la réglementation (nationale et/ou communautaire) qu'au niveau du contenu de ladite réglementation.

L'internet incarne les temps modernes numériques. L'homme l'utilise déjà pour le meilleur et pour le pire. Outil d'émancipation, il est également un outil d'asservissement. Les défis à venir pour les législateurs et les juges sont immenses, mais les réponses à apporter se fondent en réalité sur des principes d'un classicisme à toute épreuve: parvenir à ce que le droit puisse s'adapter avec pertinence aux découvertes technologiques incessantes et trouver un juste équilibre entre la sauvegarde (nécessaire) de l'intérêt général et la protection (effective) des droits individuels.

54. Le Monde, 27 février 2009. 


\section{$R E S U M E$}

La France -à l'instar de tous les autres pays - est saisie par les nouvelles technologies essentiellement incarnées par l'internet au point d'avoir jugé utile de créer un secrétariat d'État chargé de la prospective et du développement de l'économie numérique. Outil d'émancipation qui permet notamment un accès démultiplié au savoir, il est aussi un outil tyrannique qui met à mal les droits et libertés des individus. Dans des proportions variables et de manière différente, ce sont tout à la fois la vie privée, la liberté d'expression, les droits de propriété intellectuelle qui sont aux prises avec la fulgurance $d u$ développement des nouvelles technologies. 


\section{L'état des libertés}

\section{SOMMAIRE}

LIBERTÉS : UNE ÉVOLUTION PARADOXALE

LA BANALISATION DU CONCEPT DE " CENSURE 》

IMMIGRATION ET LIBERTÉS

RÉPRESSION PÉNALE ET LIBERTÉS

LES NOUVELLES TECHNOLOGIES

LES LOIS DOMESTIQUES

SANTÉ ET LIBERTÉ

LE JUGE, GARDIEN DES LIBERTÉS ?

LES LIBERTÉS DANS L'ENTREPRISE

RENOUVELER LES LIBERTÉS ?

CHRONIQUES

LA PASSATION DES POUVOIRS PRÉSIDENTIELS AUX ÉTATS-UNIS

REPÈRES ÉTRANGERS (1'r JANVIER-31 MARS 2009)

CHRONIQUE CONSTITUTIONNELLE FRANÇAISE

(1 ${ }^{\text {er }}$ JANVIER-30 AVRIL 2009)

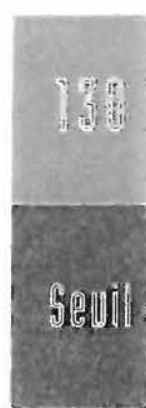

www.editionsduseuil.fr
Guy Carcassonne

Pascal Mbongo

Serge Slama

Pierre-Olivier Sur

Laurence Burgorgue-

Larsen

Nicolas Molfessis

Didier Tabuteau

Dany Cohen

Jean-Emmanuel Ray

Denys de Béchillon

Stéphanie Gruet

Pierre Astié

Dominique Breillat et Céline Lageot

Pierre Avril et Jean Gicquel 